\title{
Docencia universitaria en medio del Covid-19 \\ Adaptación y desarrollo de un curso de Bibliotecología en la virtualidad
}

\section{( Daniela Rodríguez Valerio \\ Universidad de Costa Rica. Escuela de Bibliotecología y Ciencias de la Información | daniela.rodriguezvalerio@ucr.ac.cr / https://orcid.org/oooo-0003-3737-8851}

\begin{abstract}
Resumen
El presente artículo expone el proceso de adaptación del curso "Técnicas de investigación Bibliográfica" para ser ofrecido en modalidad virtual durante el Primer Ciclo 2020 a raíz de la pandemia originada por el Covid-19. Este es un curso dirigido a estudiantes de primer año de Bibliotecología que habitualmente se ofrece de forma presencial. En primer lugar se realizó un reconocimiento de las características, contexto y posibilidades de los estudiantes participantes, posteriormente se diseñaron y evaluaron las estrategias didácticas desarrolladas por la docente. Se presentan resultados exitosos que pueden mantenerse en el curso o incluso replicarse en otros aún cuando se retomen las clases presenciales.
\end{abstract}

University teaching in the midst of Covid-19: Adaptation and development of a virtual Library Science course

\begin{abstract}
This article presents the adaptation process of the course "Bibliographic Research Techniques" to be offered in virtual mode during the first cycle 2020 as a result of the pandemic caused by Covid-19. This is a course for first-year Library and Information Sciences students that is usually offered in person. Firstly, a recognition of the characteristics, context and possibilities of the participating students was made, later the didactic strategies developed by the teacher were designed and evaluated. Successful results are presented that can be kept in the course or even replicated in others in a face-to-face course.
\end{abstract}

\section{Palabras clave}

Estrategias didácticas Educación Bibliotecología Aprendizaje virtual Covid-19

\section{Keywords}

Didactic strategies

Education

Library and Information Sciences Virtual learning

Covid-19 


\section{Introducción}

El 2020 fue un año atípico para todos los sectores de la sociedad ya que el mundo vivió una situación sin precedentes originada a raíz del Covid-19, una enfermedad infecciosa que se convirtió en pandemia. Este virus prácticamente paralizó las actividades económicas, ocasionando desempleo y aumentando las desigualdades sociales, pero también tuvo un fuerte impacto en la educación y en todos sus niveles.

Muy particularmente en el sector educativo las prácticas docentes debieron innovarse y adaptarse de forma inesperada y con poca planificación apoyándose en el diseño y aplicación de estrategias didácticas que favorecieran el aprendizaje virtual y telepresencial. Al mismo tiempo, muchos docentes tuvieron que implicarse en procesos de capacitación y, por otra parte, los estudiantes también tuvieron que aplicar o desarrollar destrezas respecto a las TIC, muchas veces en escenarios con limitantes tecnológicas y sentimientos de ansiedad, lo cual aumentó los desafíos aportados por esta pandemia.

En el caso de Costa Rica y de acuerdo al Ministerio de Salud (2020), el primer caso confirmado por Covid-19 sucedió el 6 de marzo de 2020, por lo tanto, fue a partir de esta fecha cuando las diferentes autoridades comenzaron a aplicar medidas para evitar aglomeraciones, lo cual implicó el cierre de escuelas, colegios, universidades y otros centros educativos, trasladando la educación presencial a la virtualidad.

La Universidad de Costa Rica no fue ajena a esta situación, por lo tanto, durante la primera semana del Primer Ciclo 2020 las autoridades universitarias decidieron suspender las clases presenciales, específicamente a partir del 16 de marzo (Vindas, 2020a). Esta situación implicó que se tomaran una serie de medidas, entre ellas: se tuvo que aumentar la capacidad y desempeño de la plataforma oficial para impartir clases virtuales (desde 2007 la universidad cuenta con una plataforma virtual y una Unidad de Apoyo a la Docencia Mediada con Tecnologías de la Información y Comunicación), los docentes iniciaron procesos de replanteamiento de sus cursos y capacitación, la universidad desarrolló estrategias para dotar a docentes y estudiantes de equipo y conectividad, además las autoridades generaron lineamientos para dar continuidad a las labores administrativas, académicas y de acción social.

Por lo tanto, el presente artículo tiene como objetivo general exponer las estrategias desarrolladas para ofrecer el curso "BI-1001 Técnicas de investigación bibliográfica" en modalidad virtual a razón de la pandemia originada por el Covid-19. Adicionalmente se plantearon tres objetivos específicos:

》 Reconocer el perfil y condiciones de los estudiantes de los grupos 001 y 004 del curso $\mathrm{Bl}-1001$ Técnicas de investigación bibliográfica.

» Diseñar estrategias didácticas y de evaluación virtuales acordes al perfil y situación de los estudiantes.

»Evaluar el impacto de las estrategias y sus implicaciones en el aprendizaje.

El curso en mención forma parte de los planes de estudio ofrecidos por la Escuela de Bibliotecología y Ciencias de la Información de la Universidad de Costa Rica. Habitualmente se ofrece de forma presencial a estudiantes de primer ingreso a la carrera y su objetivo es: "Proporcionar estrategias necesarias para realizar un proceso de indagación orientado por medio de técnicas de investigación bibliográfica, con el objetivo de plantear y realizar trabajos de investigación académica" (Escuela de Bibliotecología y Ciencias de la Información [EBCI], 2020). 


\section{Referentes teóricos}

\subsection{Covid-19 y su impacto en la educación superior}

La pandemia ocasionada por el Covid-19 obligó a la comunidad académica a explorar nuevas formas de enseñar y aprender, además de acuerdo a la UNESCO más de 1500 millones de estudiantes de 165 países no pudieron asistir a los centros educativos debido a esta situación (La Organización de las Naciones Unidas para la Educación, la Ciencia y la Cultura [UNESCO], 2020).

Respecto al ámbito universitario específicamente, las medidas para promover el distanciamiento social crearon un proceso de digitalización forzada, lo cual coaccionó los mecanismos de pedagogía a los de la teleeducación con el objetivo de garantizar la sostenibilidad de las clases y las labores académicas y administrativas de las instituciones de educación superior. Esta coyuntura incluso reunió a los rectores de universidades líderes de América Latina con el objeto de discutir acerca de los retos y desafíos en las universidades, acciones para generar soluciones ante la emergencia y estrategias para incentivar la transformación digital (Banco Interamericano de Desarrollo [BID], 2020).

Por lo tanto, entre los desafíos que enfrentaron las universidades ante la pandemia el BID (2020), OREALC/UNESCO Santiago (2020) y el Instituto Internacional de la UNESCO para la Educación Superior en América Latina y el Caribe (2020) destacan:

" Tuvieron que digitalizarse de forma forzada, acelerada y sin planificación previa, lo cual incidió directamente en sus procesos de comunicación efectiva al iniciar la emergencia sanitaria.

»Vivieron un desbalance en la implementación de recursos: aquellas universidades que ya tenían camino recorrido en los procesos de digitalización pudieron dar una respuesta satisfactoria a la situación, mientras que aquellas que se vieron obligadas a incursionar vieron comprometidos sus sistemas educativos y la formación de sus estudiantes.

»Se experimentó la carencia de instrumentos de evaluación en un contexto de enseñanza virtual, así como de normativa y legalidad que respaldara los procesos de evaluación del aprendizaje.

» Pocos profesores estuvieron capacitados para la teleeducación, lo cual afectó la pedagogía virtual. Esto evidenció la necesidad de los docentes de capacitarse en este tema y la importancia de la acreditación.

"Se vivieron las consecuencias de la brecha digital y el acceso limitado a tecnologías, lo cual incrementó la tasa de abandono entre los estudiantes.

» Muchos estudiantes se vieron afectados por los efectos psicológicos del confinamiento, incidiendo en su capacidad de aprendizaje.

»Se comprometió la capacidad de investigación de las universidades, debido a que estos procesos requieren de presencialidad.

»Se puso en riesgo la sostenibilidad financiera de las instituciones de educación superior, ya que muchos estudiantes vieron comprometida su capacidad de pago y en el caso de las universidades públicas estas se vieron afectadas por los planes de recuperación económica de los gobiernos.

A pesar de estos desafíos, se destaca que con ellos se han abierto oportunidades para continuar desarrollando la educación virtual en el futuro, se ha experimentado una mayor apertura de los docentes ante el cambio, se ha exaltado la importancia de la colaboración y los procesos de digitalización universitaria se han implantado. 


\subsection{El caso de la Universidad de Costa Rica y la Escuela de Bibliotecología y Ciencias de la Información}

De acuerdo a Vindas (2020a) la Universidad de Costa Rica decidió suspender oficialmente las clases presenciales a partir del 16 de marzo de 2020 como respuesta ante las medidas implantadas por las autoridades gubernamentales para garantizar el distanciamiento social. Sin embargo, desde la semana anterior (9 al 13 de marzo) se dieron instrucciones para evitar aglomeraciones, lo cual obligó a muchas unidades académicas a suspender las lecciones presenciales previo al 16 de marzo.

Se resalta que el Primer Ciclo lectivo 2020 inició en la semana del 9 al 13 de marzo, por lo tanto, muchos docentes y estudiantes no tuvieron incluso la oportunidad de encontrarse y conocerse presencialmente. Ante esta situación, la Escuela de Bibliotecología y Ciencias de la Información (EBCI) se apegó a lo estipulado por las autoridades e incentivó al personal docente a comunicarse con sus estudiantes a través de la plataforma oficial de educación virtual de la Universidad de Costa Rica, además se utilizaron las redes sociales de la Universidad, de la EBCI y el correo institucional para comunicar instrucciones y directrices a la comunidad académica y estudiantil.

Inicialmente se experimentaron problemas de comunicación entre docentes-estudiantes propios de la coyuntura, además si bien es cierto que la Universidad ya contaba con plataforma virtual y experiencia en docencia mediada por TIC, a nivel institucional se vivieron fallos en las plataformas tecnológicas debido a que se aumentó su uso y se sobrepasó su capacidad. Asimismo se vivieron sentimientos de ansiedad y preocupación por la situación sanitaria y por la adaptación inesperada de los cursos a la modalidad virtual, se tuvieron además problemas de acceso a tecnología y conectividad en algunos casos, tanto de parte de estudiantes como de docentes.

Ante este panorama, la Universidad robusteció sus plataformas tecnológicas, se iniciaron procesos de capacitación docente y desde las vicerrectorías de docencia e investigación se crearon lineamientos como los estipulados en la Resolución VD-11489-2020 "Lineamientos para la planificación, orientación e implementación de la actividad docente durante el segundo ciclo lectivo en la Universidad de Costa Rica", en la Resolución VD-11502-2020 "Lineamientos académicos y administrativos para la docencia con componente virtual", y la "Guía para la defensa pública virtual de trabajos finales de graduación de grado", entre otros.

Desde la Escuela de Bibliotecología y Ciencias de la Información se idearon estrategias de comunicación con la comunidad estudiantil, docente y administrativa, como por ejemplo un uso más intensivo de las redes sociales como Facebook y Youtube; además se incursionó en la aplicación de mensajería instantánea WhatsApp y la red social Instagram. Asimismo, la EBCI participó de la elaboración de la Guía para Defensas Virtuales de la Universidad de Costa Rica (Vindas, 2020b), se motivó a los docentes a participar de experiencias de capacitación y la Escuela se organizó para prestar equipo tecnológico a sus estudiantes y docentes en desventaja. Todas estas iniciativas representaron esfuerzos importantes para que el Primer Ciclo 2020 pudiera culminar satisfactoriamente y se contara con mejores herramientas y conocimientos para afrontar el Segundo Ciclo 2020.

\subsection{Virtualización de cursos}

La pandemia originada por el Covid-19 obligó a las instituciones de educación superior a utilizar sus recursos con creatividad para promover la virtualización total de sus cursos, a pesar de que muchos de estos estuvieran planificados para ofrecerse exclusivamente en la presencialidad. Sin embargo, a pesar de que a muchas instituciones educativas les tomó esfuerzos importantes adaptarse a la educación virtual, es 
importante destacar que esta ha sido una tendencia que se ha venido estableciendo en el ámbito educativo desde años atrás. Al respecto, Moreira-Segura y DelgadilloEspinoza (2015) explican que esta modalidad de educación ha sido posible por el auge de las nuevas tecnologías de información y comunicación (NTIC), las cuales han posibilitado el rompimiento de las brechas de distancia, aminorar la rigidez de los horarios y la adaptación a los estilos de vida modernos.

Ahora bien, los procesos de virtualización de cursos requieren de consideraciones importantes para que se puedan desarrollar de la mejor manera, entre ellas:

》 Idear protocolos para favorecer una comunicación fluida y asertiva.

"Es necesario brindar un acompañamiento constante a las actividades que ejecutan los participantes.

»Deben elegirse apropiadamente los recursos tecnológicos que mediarán la enseñanza.

»En el hecho pedagógico, debe procurarse brindar respuestas rápidas a las dudas de los estudiantes.

»Implementar indicaciones con claridad.

»El docente debe tener presencia en el aula virtual, es decir, debe evitar presentarse de forma esporádica o nula.

" Mostrar cercanía con los estudiantes.

"Evitar sobrecargar a los educandos.

"Fomentar la interacción y la colaboración (Moreira-Segura y DelgadilloEspinoza, 2015).

Asimismo, durante el contexto de pandemia la Vicerrectoría de Docencia de la Universidad de Costa Rica emitió una serie de "Lineamientos académicos y administrativos para la docencia con componente virtual", entre los que se destacan:

» Mediación del aprendizaje a través de una plataforma educativa oficial de la institución.

》Propiciar las interacciones docente-estudiante, estudiante-estudiante.

" Incorporar modalidades innovadoras de docencia.

"Fomentar la igualdad de oportunidades y la inclusión.

» Alternar clases sincrónicas con asincrónicas, donde las sincrónicas no sobrepasen los 50 minutos.

»En las clases sincrónicas no evaluadas, brindar al estudiante la libertad de utilizar o no su audio y video.

"Manejar con discreción las grabaciones de las clases, ya que las mismas contienen datos personales de los datos e información que no es pública.

»Aplicar la innovación evaluativa y tener flexibilidad ante situaciones no previstas, entre otros (Universidad de Costa Rica. Vicerrectoría de Docencia, 2020).

\subsection{Acerca del curso "Técnicas de investigación bibliográfica"}

Según se explica en el programa del curso BI-1001 "Técnicas de investigación bibliográfica" ofrecido durante el Primer Ciclo del año 2020,

Este es un curso orientado a proporcionar al estudiantado de las carreras de Bachillerato en Bibliotecología con énfasis en Ciencias de la Información y Bachillerato en Bibliotecología con énfasis en Bibliotecas Educativas, los conocimientos necesarios para la utilización de técnicas de investigación bibliográfica y presentación de informes, en miras a su aplicación en trabajos académicos y de investigación que serán desarrollados a lo largo de la carrera (Escuela de Bibliotecología y Ciencias de la Información, 2020: 2). 
Es además un curso donde se le enseña al estudiantado a incursionar en procesos de investigación, a discriminar y utilizar fuentes de información confiables, a redactar y presentar informes y a utilizar normas para la citación bibliográfica, entre otros. Asimismo, puede ofrecerse en modalidad bajo virtual, es decir, el $75 \%$ del mismo se abarca de forma presencial y el $25 \%$ puede ser desarrollado de forma virtual por el docente a través de la plataforma educativa oficial (Universidad de Costa Rica. Vicerrectoría de Docencia, 2020).

Respecto a sus contenidos, entre ellos se destacan:

» Fuentes primarias, secundarias y terciarias en formato impreso y digital.

"Servicios y recursos en bibliotecas públicas, públicas municipales, escolares, universitarias, especializadas, biblioteca nacional, centros de documentación y archivos.

»Uso de catálogos en línea y de colecciones de las bibliotecas.

» Evaluación crítica de fuentes de información.

" Normativa de la UCR respecto al plagio.

»Estilos internacionales para la citación.

»Software para la citación bibliográfica.

»Etapas de la investigación bibliográfica.

"Uso de diccionarios para la redacción.

" Partes de un trabajo académico y su presentación.

» Nociones básicas sobre la exposición oral.

"Elaboración de presentaciones (Escuela de Bibliotecología y Ciencias de la Información [EBCl], 2020).

Es importante indicar además que este curso se ofrece a estudiantes de nuevo ingreso a la carrera de Bibliotecología, por lo tanto, los grupos suelen ser grandes (aproximadamente 30 personas, el cupo es de hasta 32 por la capacidad de las aulas) e integrados mayoritariamente por estudiantes que recién finalizan sus estudios de secundaria (jóvenes de 17-18 años). Sin embargo, también es usual que lo matriculen estudiantes de años avanzados o que no son de primer ingreso pues existen casos en que se trasladan de carrera o que deciden retomar sus estudios a edades más avanzadas. No obstante, se resalta que los grupos suelen estar integrados principalmente por estudiantes que recién se integran a la vida universitaria.

En cuanto a las estrategias didácticas que suelen ser aplicadas por los docentes se destacan aquellas que implican una alta presencialidad, por ejemplo, el trabajo colaborativo, discusiones en clase, visitas guiadas a bibliotecas, plenarias con expertos invitados, entre otras.

Ahora bien, durante el Primer Ciclo 2020 este curso coincidió con la pandemia originada por el Covid-19, por lo tanto, el mismo tuvo que adaptarse y adecuarse a la virtualidad, lo cual implicó un replanteamiento de sus estrategias didácticas y de mediación con los estudiantes. Para estos efectos se consideraron los "Lineamientos académicos y administrativos para la docencia con componente virtual" desarrollados por la Vicerrectoría de Docencia de la Universidad de Costa Rica, así como otras directrices y recomendaciones que se fueron instrumentando o facilitando en la Universidad y dirigidas a su personal docente. 


\section{Metodología}

A continuación se exponen el tipo de investigación, enfoque, población en estudio, técnica de recolección de información y, se describen cada una de las etapas que se llevaron a cabo para el desarrollo de la investigación:

\subsection{Tipo de investigación y enfoque}

Se desarrolló una investigación exploratoria con enfoque cuantitativo. Al respecto los estudios exploratorios "sirven para preparar el terreno y generalmente anteceden a investigaciones con alcance descriptivo, correlacionales o explicativos" (HernándezSampieri y Mendoza Torres, 2018: 106). Además, esta clase de estudios se emplean cuando se desea examinar un fenómeno o problema de investigación nuevo o poco estudiado sobre el cual se tienen muchas dudas o no se ha abordado antes, o bien, si se desea indagar los temas desde diferentes perspectivas.

La investigación tiene una orientación cuantitativa, ya que los datos se encuentran en forma de números, su recolección se fundamentó en la medición de variables y los datos se analizaron con métodos estadísticos (Hernández-Sampieri y Mendoza Torres, 2018).

\subsection{Población en estudio}

Se trabajó con 56 estudiantes matriculados en los grupos 01 y 04 del curso BI-1001 Técnicas de investigación bibliográfica durante el Primer Ciclo 2020, el cual se extendió desde marzo a julio del año 2020. La mayoría tienen de 17 a 21 años, son de primer ingreso a la universidad y están matriculados en las carreras de bachillerato en Bibliotecología con énfasis en Ciencias de la Información y bachillerato en Bibliotecología con énfasis en bibliotecas educativas.

A ellos se les aplicó un cuestionario al inicio del curso con el objetivo de conocer su contexto, posibilidades y expectativas respecto a la virtualización sorpresiva del curso, posteriormente se les aplicó otro cuestionario al finalizar el ciclo lectivo, el cual buscó evaluar las estrategias didácticas desarrolladas por la docente a cargo de ambos grupos.

\subsection{Técnica de recolección}

Al iniciar el curso se aplicó un cuestionario en línea para recolectar información sobre los estudiantes (Apéndice 1) dado que la emergencia sanitaria no le permitió a la docente reunirse con una parte de ellos, y con quienes sí tuvo posibilidad de reunirse fue posible tener únicamente una clase presencial. Este cuestionario se organizó en 5 secciones que buscaron indagar sobre:

1. Información general de los estudiantes

2. Condiciones tecnológicas y ambientales de los estudiantes

3. Circunstancias de aprendizaje de los estudiantes

4. Expectativas de los estudiantes respecto al curso

5. Retos de los estudiantes y sugerencias para el curso ante el Covid-19

Es importante mencionar que este cuestionario inicial fue resuelto por 42 de los estudiantes, lo cual representa el $75 \%$ de la población.

Posteriormente, al finalizar el curso se aplicó otro cuestionario en línea (Apéndice 2) para evaluar el desarrollo del curso y el impacto de las estrategias didácticas 
implementadas por la docente. Este instrumento se organizó en 3 secciones que buscaron indagar la opinión de los estudiantes sobre:

1. Estrategias didácticas desarrolladas por la docente

2. Estrategias de evaluación desarrolladas por la docente

3. Opinión general del curso

Este cuestionario fue resuelto por 36 estudiantes, lo cual representa el $64.2 \%$ de la población.

Es importante indicar que en la Universidad de Costa Rica se cuenta con un Comité Ético Científico que regula las investigaciones con seres humanos, por lo tanto, siguiendo sus directrices se elaboró un consentimiento informado para invitar a los estudiantes a participar de la investigación e informales sobre sus implicaciones (Apéndice 3).

\subsection{Adaptación y desarrollo del curso "Técnicas de investigación bibliográfica”}

\subsubsection{Etapa 1: Caracterización de los estudiantes y su contexto}

Para esta etapa, la docente diseñó un instrumento para conocer a los estudiantes, su contexto y posibilidades, dado que no pudo encontrarse de forma presencial con la mayoría de ellos; además les explicó que esta información se recolectaría de forma confidencial, voluntaria y para fines académicos. Por lo tanto, construyó un formulario en línea que compartió en el aula virtual y en un grupo cerrado de WhatsApp integrado por estudiantes y docente.

Para el diseño de las preguntas de este instrumento se consultó bibliografía acerca de la virtualización de cursos, por lo tanto se indagó sobre su sexo, edad, procedencia (rural o urbana), dificultades motoras del estudiante, destrezas tecnológicas, condiciones ambientales y tecnológicas, disponibilidad de conectividad, necesidades respecto al aprendizaje en la virtualidad, apertura a las clases sincrónicas, actitud hacia el trabajo en grupo, retos durante la pandemia relacionados con el proceso educativo, expectativas y recomendaciones para el curso. Para recolectar esta información se estableció una fecha límite con el objetivo de continuar con la siguiente etapa.

\subsubsection{Etapa 2: Diseño de las estrategias didácticas del curso.}

Para el cumplimiento de esta etapa la docente revisó la información suministrada por los estudiantes en la etapa 1. Por lo tanto, diseñó estrategias didácticas y de evaluación adecuadas a la situación general de los estudiantes. Las mismas se planearon y construyeron con base en su experiencia docente y en los conocimientos adquiridos durante capacitaciones en línea y webinars organizados por la Unidad de Apoyo a la Docencia Mediada con Tecnologías de la Información y la Comunicación (METICS) de la Universidad de Costa Rica, también se tomaron en cuenta los "Lineamientos académicos y administrativos para la docencia con componente virtual", entre otras directrices que se fueron incorporando durante la pandemia.

\subsubsection{Etapa 3: Evaluación de las estrategias didácticas y de evaluación del curso}

Esta etapa de la investigación se llevó a cabo al finalizar el curso, por lo tanto, la docente compartió otro formulario en línea en el cual consultó a los estudiantes acerca de su aprendizaje y las estrategias implementadas por la docente para desarrollar el curso. Asimismo, se indagó su opinión sobre el mismo, aciertos y desaciertos. 


\section{Presentación y análisis de resultados}

En los siguientes párrafos se muestran los resultados de cada una de las etapas de la investigación, así como un posterior análisis de la información presentada:

\subsection{Etapa 1: Caracterización de los estudiantes y su contexto}

La mayoría de los estudiantes matriculados en el curso durante el Primer Ciclo 2020 (37 estudiantes) tienen edades que oscilan entre los 17 a 21 años, lo cual indica que son de primer ingreso a la Universidad o que cursan los primeros años de otra carrera. Se destaca además que 28 de ellos provienen de zonas urbanas y 14 de zonas rurales. La mayoría de ellos no presenta alguna dificultad auditiva o motora que le afecte el realizar sus clases virtuales, sin embargo 6 de ellos manifestaron presentar dificultades visuales o relacionadas con afectaciones desarrolladas al exponerse a las pantallas y letras pequeñas de los dispositivos móviles.

En cuanto a sus destrezas respecto al manejo de la tecnología, solamente 2 de los estudiantes participantes expresaron que "no son diestros".

Respecto a la disponibilidad de equipo, la mayoría manifestó que disponía de una computadora para participar de las clases en el horario del curso, mientras que 4 de ellos manifestaron que no y que sólo disponían de un teléfono ( 3 de ellos con contrato telefónico post-pago y 1 con pre-pago).

Relacionado con acceso a conectividad, el 73.8\% expresó que disponía de una buena conexión a internet.

Ahora bien, respecto a condiciones ambientales, 28 de los estudiantes manifestaron disponer de un buen ambiente para realizar sus clases virtuales, mientras que 14 de ellos no.

En cuanto a situaciones de didáctica y aprendizaje propiamente, la mayoría de los estudiantes expresó preferir una combinación de clases sincrónicas y asincrónicas (80.9\%) y un $19 \%$ se inclinó por las asincrónicas, esto coincide con la investigación de Kikut (2020). Se destaca que un o\% manifestó preferir un curso desarrollado únicamente con clases sincrónicas. Asimismo la mayoría se inclinaron por trabajar solos y no en grupo (posiblemente porque la mayoría son de primer ingreso y no se conocen).

Finalmente, ante la pregunta ¿Qué características debe tener un curso virtual para que usted pueda aprender?, los estudiantes respondieron (Cuadro 1):

Cuadro 1: Características que debe tener un curso virtual para posibilitar el aprendizaje

\begin{tabular}{|l|c|}
\hline Característica & Frecuencia \\
\hline Debo tener buena comunicación con la docente & 37 \\
\hline Me funciona acceder a variedad de material didáctico: videos, lecturas y audio & 36 \\
\hline Necesito tener indicaciones claras por escrito & 35 \\
\hline Necesito realizar actividades prácticas & 26 \\
\hline Necesito que las herramientas y sistemas empleados sean fáciles de utilizar & 24 \\
\hline Otras & 4 \\
\hline
\end{tabular}

Fuente: Elaboración propia, 2020 


\subsection{Etapa 2: Diseño de las estrategias didácticas del curso.}

Partiendo de la información recolectada en la etapa 1 la docente planificó estrategias didácticas y de evaluación que fomentaron el trabajo individual, además consideró que una parte de los estudiantes estaban viviendo la brecha tecnológica. Por lo tanto, el curso se desarrolló aplicando las siguientes estrategias:

1. Se creó un grupo cerrado en WhatsApp Business integrado por la docente y los estudiantes. El mismo se moderó durante el ciclo y se les permitió escribir únicamente durante el horario y día del curso. Los estudiantes que tenían dudas fuera del horario podían escribir aparte a la profesora. Este grupo fue muy importante para mantener una comunicación constante entre ambas partes.

2. Para apoyar el curso se utilizaron 17 videos grabados por la profesora, material escrito complementario en formato digital y audios. Todos estos recursos se compartieron mediante el aula virtual de la Universidad y WhatsApp Business.

3. El curso se desarrolló primordialmente a través de clases asincrónicas que fueron grabadas previamente por la profesora con ayuda del software OBS. Las mismas se publicaron en Youtube y los enlaces se compartieron en el aula virtual oficial de la Universidad de Costa Rica.

4. Se organizaron pocas clases sincrónicas utilizando Zoom, únicamente para aclarar dudas, ver temas específicos y mantener un contacto más personal con los estudiantes, las mismas se desarrollaron en el horario del curso con recesos cada 50 minutos. Es importante detallar que previo a las clases sincrónicas se redactó un manual de la herramienta Zoom, el cual fue validado por un estudiante del curso antes de compartirlo con los demás participantes.

5. Se utilizaron formularios de Google Forms para diseñar cuestionarios de evaluación con preguntas abiertas y cerradas.

6. Se crearon algunas prácticas opcionales para que los estudiantes pudieran realizarlas previo a las evaluadas.

7. Cada una de las evaluaciones se acompañó de un archivo con instrucciones detalladas escritas y una rúbrica para calificación.

8. Se organizaron algunos foros de discusión sobre temas del curso.

9. Se realizaron trabajos prácticos con instrucciones claras y exhaustivas, los mismos se acompañaron de videos grabados por la profesora, además los estudiantes tuvieron una semana para desarrollarlos y entregarlos a través del aula virtual oficial.

10.Los trabajos prácticos se diseñaron de modo que los estudiantes pudieran trabajarlos en un procesador de texto instalado en su computadora o en la nube. Los estudiantes sin acceso a computadoras utilizaron Google Docs por ejemplo.

\section{Etapa 3: Evaluación de las estrategias didácticas y de evaluación del curso}

En cuanto a las opiniones de los estudiantes respecto a las estrategias desarrolladas por la docente, en primer lugar, el 100\% manifestó que las clases pre-grabadas en combinación con algunas reuniones sincrónicas les permitió obtener un buen aprendizaje. Respecto a esto, indicaron que esta metodología les permite revisar la materia en momentos adecuados pues algunos compartían el equipo tecnológico con hermanos o experimentan problemas de conectividad por ejemplo. Asimismo, el video se puede pausar, regresar y repetir por si alguna inquietud persiste. Destacaron además que los videos pre-grabados fueron de utilidad pues disponían de buena calidad de audio y video.

En relación con las instrucciones dadas, en el gráfico 1, se aprecia que la mayoría de estudiantes evaluó las instrucciones como "muy claras", algunos de ellos expresaron que fueron "claras" y ninguno indicó que fueron "poco claras". Al respecto, los estudiantes valoraron de forma positiva que se creara una rúbrica para cada evaluación 
y que se les diera indicaciones detalladas y por escrito. Además, indicaron que les favoreció disponer del contacto de la profesora en WhatsApp pues de este modo pudieron aclarar inquietudes de forma complementaria y oral.

En cuanto al uso dado por la profesora a la aplicación WhatsApp, el 100\% opinó que fue "bueno", además se sintieron cómodos formando parte de un grupo cerrado que fue moderado durante el ciclo. De este modo se evitaron los mensajes tipo "spam" o no deseados, otorgando un uso exclusivamente didáctico a la herramienta. Este resultado coincide con los arrojados en la investigación de Rodríguez Valerio (2020).

Ahora bien, respecto a las clases sincrónicas, la mayoría de los estudiantes opinaron que fueron "buenas", 5 de ellos comentaron que fueron regulares y ninguno indicó que fueron malas, tal como se muestra en el gráfico 2.

$\mathrm{Al}$ respecto, resaltaron que fueron dinámicas, complementarias a la materia, bien planificadas y que les ayudó mucho contar con un manual de uso de la herramienta Zoom. Destacaron que la organización de recesos cada 50 minutos colaboró para evitar sentimientos de "saturación" y les hizo sentir cómodos el poder expresar sus dudas de forma oral o mediante el chat sin necesariamente tener la cámara activa. Sí hicieron la recomendación de motivar a más compañeros a participar durante las clases, porque de este modo se vuelven más interactivas.

Sobre la prontitud para recibir sus evaluaciones calificadas, la mayoría de los estudiantes expresaron que fue "muy importante" y 3 de ellos lo valoraron como "importante"; ninguno indico que resultara "poco importante", como lo muestra el gráfico 3.

Posiblemente esto les favoreció para corregir oportunamente sus errores en prácticas subsecuentes, lo cual favorece su aprendizaje.

En cuanto a las estrategias de evaluación que les ayudó a comprender mejor los contenidos del curso la mayoría opinó que fueron las prácticas creadas mediante formularios de Google, la elaboración y presentación de trabajos escritos pues se trató de un curso de técnicas de investigación; además de una serie de prácticas opcionales que la profesora asignó para que pudieran practicar previo a las que sí eran evaluadas. Respecto a los foros de aprendizaje, solamente 14 estudiantes indicaron que ayudaron a su aprendizaje.

Finalmente se les consultó sobre aciertos del curso en la virtualidad, ante lo cual resaltaron que los videos fueran diseñados por la docente, que se les entregara sus trabajos calificados a tiempo, que se hicieran evaluaciones calificadas y no calificadas de forma constante, que se atendieran sus dudas de forma rápida y práctica. Respecto a desaciertos, resaltaron algunos inconvenientes técnicos que se presentaron durante las clases sincrónicas, la imposibilidad de realizar giras, trabajo de campo y que algunos compañeros no pudieran conectarse para participar de las sesiones.

\section{Conclusiones}

Al concluir esta investigación se destaca la importancia de considerar que el hecho pedagógico responde a públicos y contextos diferentes, por lo tanto es responsabilidad del docente tomar en cuenta las características y necesidades particulares de sus estudiantes para planificar estrategias didácticas y de evaluación significativas, especialmente cuando viven sentimientos de ansiedad, brecha digital o no disponen de igualdad de condiciones al momento de someterse al proceso de enseñanzaaprendizaje. 
Gráfico 1. Opinión sobre las instrucciones dadas por la profesora para desarrollar las clases

Gráfico 2. Opinión sobre las clases sincrónicas

Gráfico 3. Para usted ¿Qué tan importante fue recibir sus evaluaciones calificadas con prontitud?

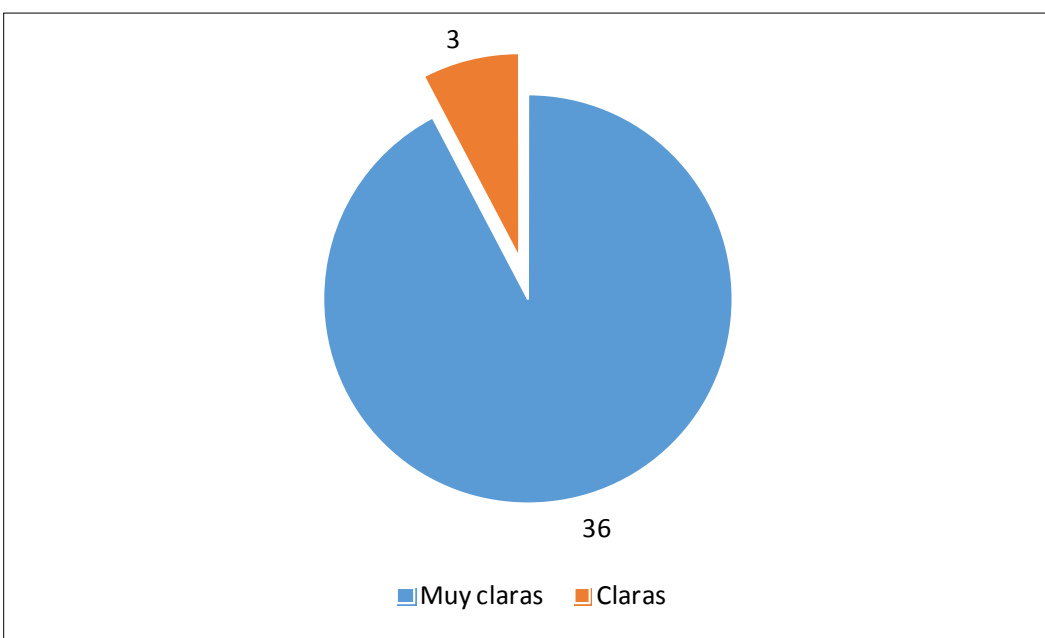

Fuente: Elaboración propia, 2020

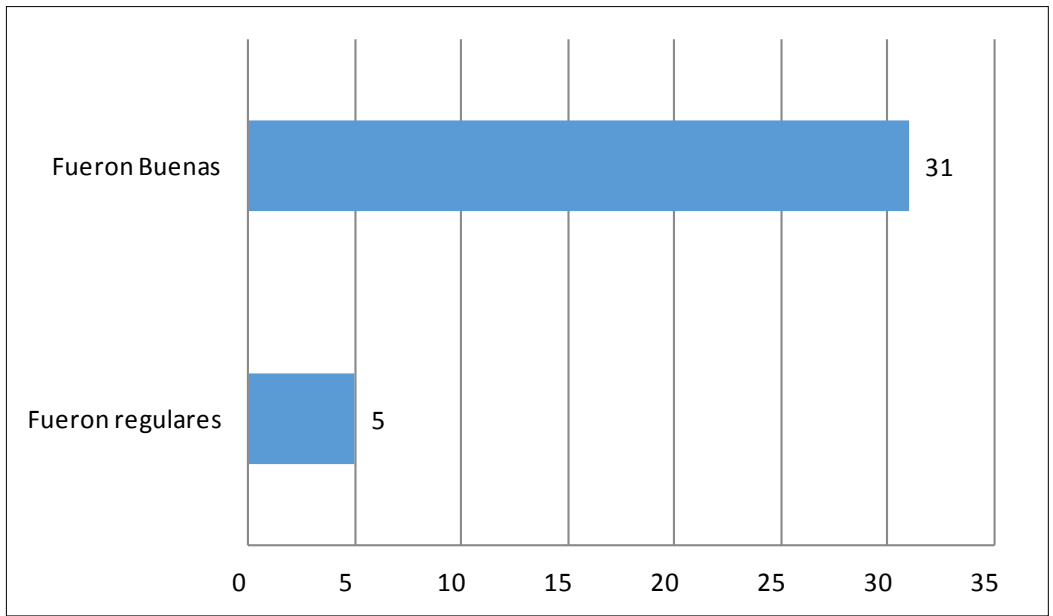

Fuente: Elaboración propia, 2020

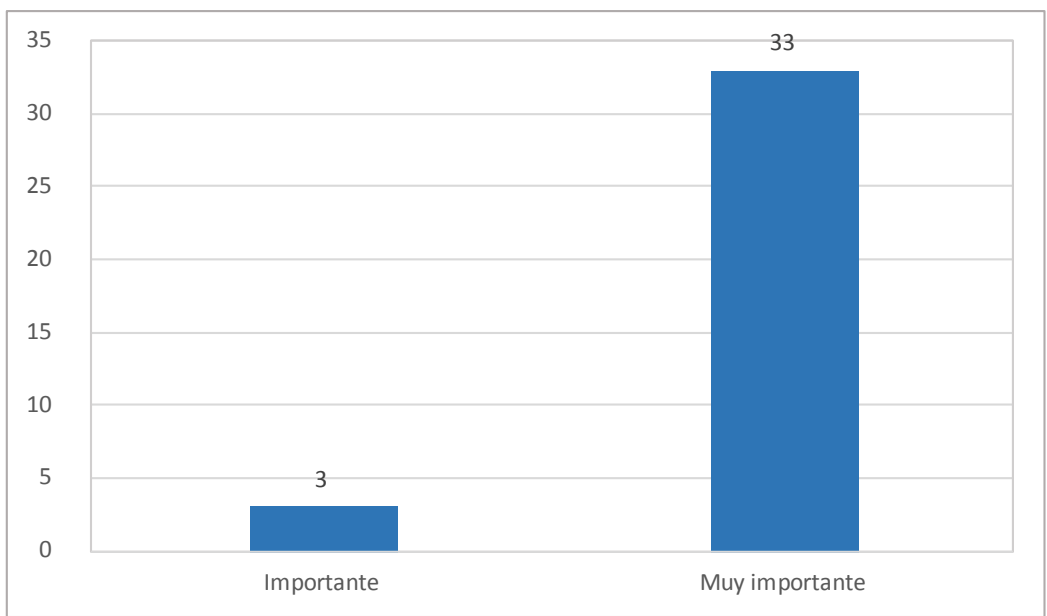


Asimismo, es relevante mencionar que los estudiantes jóvenes poseen importantes destrezas respecto al uso de la tecnología; sin embargo, también requieren de apoyos por parte del docente para hacer un uso óptimo de la misma en un contexto educativo virtual. Por lo tanto, el uso de manuales, plataformas o herramientas intuitivas es fundamental para favorecer una actitud positiva hacia la materia y el aprendizaje.

En un ambiente de virtualidad es muy relevante una constante comunicación entre el docente y sus estudiantes ya que esta favorece la confianza y ayuda a mitigar los sentimientos de miedo o frustración ante la materia o el contexto que se vive. Para promover esta comunicación lo más conveniente es utilizar los medios que usan los estudiantes, siempre procurando establecer comunicación oral y escrita.

En cuanto a las estrategias didácticas desarrolladas en la virtualidad, se considera que el trabajo práctico debe mantenerse pues promueve un aprendizaje significativo y los estudiantes lo valoran positivamente ya que les permite aplicar el conocimiento. De igual forma, el docente debe combinar métodos sincrónicos con asincrónicos, además de apoyar las lecciones con material escrito y audiovisual idealmente elaborado por sí mismo.

Respecto a las estrategias de evaluación en la virtualidad se concibe como buena práctica el asignar evaluaciones optativas y sin calificación que les permitan a los estudiantes familiarizarse con las plataformas o herramientas tecnológicas y además entrar en confianza con las metodologías, especialmente durante la pandemia Covid19 que originó un contexto inesperado. Esta estrategia les permite a los estudiantes además reducir la ansiedad ante las evaluaciones.

Finalmente, se rescata que la pandemia Covid-19 les permitió a los docentes diversificar sus conocimientos y estrategias de enseñanza lo cual resulta favorecedor para el presente y futuro de la educación. De ahora en adelante las tecnologías bien empleadas y justificadas podrán continuar utilizándose como un medio que posibilita el aprendizaje a distancia, reduciendo brechas de tiempo y espacio.

\section{Agradecimientos}

Se agradece a los estudiantes del curso BI-1001 Técnicas de Investigación Bibliográfica, grupos 004 y 002 por participar de esta investigación y aportar su información y opiniones respecto a las estrategias didácticas y de evaluación desarrolladas en un contexto atípico de pandemia. Este aporte permite retroalimentar futuros cursos y favorece el quehacer de la Escuela de Bibliotecología y Ciencias de la Información de la Universidad de Costa Rica. 


\section{Q Apéndices}

\section{Apéndice 1}

\section{Información de los estudiantes}

Este instrumento tiene como objetivo reconocer las características de los estudiantes matriculados en el curso "Técnicas de Investigación bibliográfica" durante el I ciclo 2020, asimismo busca conocer cómo es su contexto, posibilidades y expectativas respecto a la virtualización del curso. La información suministrada se utilizará para fines académicos, de investigación y las respuestas son anónimas.

Disponible en: https://forms.office.com/Pages/ResponsePage.aspx?id=DQSIkWdsWoyxEjajBLZtrQA AAAAAAAAAAAN_snACqgUQogWVIRCWkNHRIZXUTNVQ11dWVVZUTIZDNi4u

Información general

1.Sexo

Femenino

Maculino

Prefiero no responder

2.Edad en años cumplidos

de 17 a 21 años

de 22 a 26 años

de 27 a 31 años

32 años o más

3.¿Es usted de primer ingreso?

$\mathrm{Si}$

No

4.¿Desde qué zona realiza usted sus clases virtuales?

Desde una zona urbana

Desde una zona rural

5.¿Posee alguna dificultad visual, auditiva o motora que le esté afectando para realizar sus clases virtuales?

Sí (pasa a la siguiente pregunta)

No (pasa a la pregunta No. 7)

6.Por favor comente sobre su situación

7.¿Cómo considera que son sus destrezas respecto al uso de la tecnología?

Soy muy diestro (a)

Soy más o menos diestro (a)

No soy diestro (a)

Condiciones tecnológicas y ambientales

8.¿Posee usted una computadora para realizar sus clases virtuales en el horario del curso?

$\mathrm{Si}$

No

9.¿Dispone de una buena conexión a internet para realizar sus clases virtuales en el horario del curso?

$\mathrm{Si}$

No 
10.¿Su ambiente es favorable para realizar clases virtuales?

Para responder esta pregunta considere si posee un espacio cómodo y libre de ruido

Si

No

11.¿Es su teléfono el único medio para realizar sus clases virtuales?

Si (pasa a la siguiente pregunta)

No (pasa a la pregunta No.13)

12.¿Qué tipo de plan posee?

Postpago

Prepago

\section{Circunstancias de aprendizaje}

13. Para usted ¿qué características debe tener un curso virtual para que usted pueda aprender?

Puede marcar varias opciones

Debo tener buena comunicación con la docente

Necesito tener indicaciones claras por escrito

Necesito realizar actividades prácticas

Me funciona acceder a variedad de material didáctico: videos, lecturas y

audio por ejemplo

Necesito que las herramientas y sistemas empleados sean fáciles de usar

14. Durante esta pandemia, ¿ha tenido clases en vivo? (por plataformas como Zoom)

Sí (pasa a la siguiente pregunta)

No (pasa a la pregunta No. 16)

15.¿Qué tipo de clases virtuales le han hecho sentir más cómodo (a)?

Sincrónicas (en vivo)

Asincrónicas (puedo organizar mi tiempo y realizarlas durante la semana)

Una combinación de clases sincrónicas y asincrónicas

16.¿Estaría dispuesto (a) a trabajar en grupo de forma virtual?

$\mathrm{Si}$

No

\section{Expectativas respecto al curso}

17. ¿Cuáles son sus expectativas respecto al curso en modalidad virtual?

\section{Retos y sugerencias ante el covid-19}

18. Comente, ¿cuál ha sido su mayor reto durante la virtualización de cursos a raíz de la pandemia?

19. Finalmente ¿tiene alguna sugerencia o idea para mejorar el desarrollo del curso durante la pandemia? si es así por favor comente 


\section{Apendice 2}

\section{Evaluación del curso "Bl-1001 Técnicas de investigación bibliográfica"}

El presente instrumento tiene como objetivo conocer su opinión y experiencias respecto a las estrategias didácticas y de evaluación utilizadas en el curso "Técnicas de investigación bibliográfica" el cual fue desarrollado en modalidad virtual y atendiendo a la epidemia ocasionada por el Covid-19 durante el I ciclo 2020. Las respuestas son anónimas y se utilizarán para fines académicos, además, los resultados de esta investigación se publicarán en un artículo científico en el cuál se mencionará a los estudiantes en la sección de agradecimientos.

Disponible en: https://forms.office.com/Pages/ResponsePage.aspx?id=DQSIkWdsWoyxEjajBLZtrQA AAAAAAAAAAAN_snACq9UMUoyUk1HMUhKNFMxQoNJVkICUkNGWUsHWi4u

\section{Instrucciones}

Por favor responda de manera individual y honesta cada una de las interrogantes que se le presentan en este cuestionario

\section{Estrategias didácticas}

1. Considerando que gran parte del curso se desarrolló a través de videos pre-grabados por la profesora, ¿cómo califica su aprendizaje con esta metodología?

Bueno

Regular

Malo

2. Por favor justifique su respuesta a la pregunta anterior

3. ¿Cómo califica usted las instrucciones dadas por la profesora para el desarrollo de cada una de las lecciones en modalidad virtual?

Las instrucciones se brindaron a través de textos en cada una de las semanas de la Plataforma Mediación Virtual y se complementaron con el uso de WhatsApp

\section{Muy claras}

Claras

Poco claras

4. Justifique su respuesta a la pregunta anterior

5. Considerando que se utilizó WhatsApp desde el primer día de clases para resolver dudas, comunicar avisos y enviar material complementario, ¿cómo calificaría el uso dado a esta aplicación?

Bueno

Regular

Malo

6. ¿Se sintió cómodo (a) participando en un grupo de WhatsApp cerrado y que se habilitó únicamente durante el período de la clase?

$\mathrm{Si}$

No

7. ¿Cuál es su opinión acerca de las clases en vivo y que se desarrollaron a través de la plataforma Zoom?

Fueron Buenas

Fueron regulares

Fueron malas

8. Justifique su respuesta anterior 


\section{Estrategias de evaluación}

9. Seleccione las estrategias de evaluación que le ayudaron a comprender mejor los contenidos del curso

Puede marcar varias opciones

Prácticas con formularios de Google

Elaboración y presentación de trabajos escritos

Participación en foro de discusión

10. ¿Considera que las prácticas opcionales contribuyeron a su aprendizaje?

$\mathrm{Si}$

No

No las hice

11. Para usted, ¿qué tan importante fue recibir sus evaluaciones calificadas con prontitud?

Muy importante

Importante

Poco importante

12. ¿Cómo califica usted las instrucciones dadas por la profesora para el desarrollo de cada una de las evaluaciones y su calificación?

Muy claras

Claras

Poco claras

13. Justifique su respuesta anterior

Opinión general del curso

14. En su opinión, ¿cuáles fueron aciertos del curso en la modalidad virtual?

15. Y, ¿cuáles fueron desaciertos de este curso en la modalidad virtual?

16. ¿Está de acuerdo con que se divulgue una fotografía del grupo en un futuro artículo científico?

Si

No

17. Finalmente, ¿cómo se siente después de haber finalizado el curso?

Al inicio tenía interés y lo perdí

Al inicio no tenía interés y ahora sí

Al inicio no tenía interés y sigo sin tenerlo

$\mathrm{Al}$ inicio tenía interés y lo mantengo

Al inicio tenía interés y aumentó

18. Escriba algún otro comentario que desee expresar 


\section{Apéndice 3}

Consentimiento informado para participar en investigación

Disponible: https://forms.office.com/Pages/ResponsePage.aspx?id=DQSIkWdsWoyxEjajBLZtrQAAA

AAAAAAAAAN_snACq9URFFWRkM2TzExRVIVMzU4 $4 N_{1}$ NDNDBZTkNEQi4u

\section{Nombre de la investigadora: Prof. Daniela Rodríguez Valerio}

Los datos recopilados serán utilizados para realizar una investigación cuyo objetivo es reconocer cuáles estrategias didácticas y de evaluación son efectivas para virtualizar el curso Bl-1001 Técnicas de investigación bibliográfica a razón de la pandemia originada por el Covid-19. Con esto se generará conocimiento aplicable en la virtualización de cursos en la enseñanza de la bibliotecología. La investigación se desarrollará bajo un enfoque cuantitativo durante el I ciclo 2020 y busca implicar a un aproximado de 60 estudiantes que están matriculados en el curso. La participación en esta investigación es voluntaria y no tiene ninguna implicación en el resultado de la nota final del curso. La investigadora garantiza el estricto manejo y confidencialidad de la información, tanto durante como después del proceso de investigación cuando se publiquen los resultados en un artículo científico o conferencia internacional.

\section{¿Qué se hará?}

Para participar en la investigación, el sujeto de investigación (en este caso el estudiante) deberá participar de las clases, realizar las evaluaciones programadas para aprobar el curso y posteriormente llenará un cuestionario de evaluación de las estrategias didácticas y de evaluación empleadas por la docente durante el desarrollo del ciclo lectivo.

\section{Riesgos}

No tiene

Beneficios

La participación en esta investigación no implica un beneficio directo para el estudiante, sin embargo estará aportando al eje investigativo de la Escuela de Bibliotecología y Ciencias de la Información y de la Universidad de Costa Rica.

\section{Consentimiento}

La persona aquí firmante ha leído toda la información descrita en esta fórmula antes de firmarla. Se le ha brindado la oportunidad de hacer preguntas y estas han sido contestadas en forma adecuada. Por lo tanto, declara que entiende de qué trata la investigación, las condiciones de su participación y accede a participar como sujeto de investigación en este estudio.

1. Digite su nombre y apellidos

2. Digite su cédula 


\section{Q Referencias bibliográficas}

» Banco Interamericano de Desarrollo [BID]. 2020. La educación superior en tiempos de Covid-19: Aportes de la segunda reunión del diálogo virtual con rectores de universidades líderes de América Latina. 19-20 mayo 2020. Washington, DC: BID. $<$ https://publications.iadb.org/publications/spanish/document/La-educacion-superior-en-tiempos-de-COVID-19-Aportes-de-la-Segunda-Reunion-delDi\%C3\%A1logo-Virtual-con-Rectores-de-Universidades-Lideres-de-AmericaLatina.pdf> [Consulta: 19 diciembre 2020].

" Costa Rica. Ministerio de Salud. 2020. Caso confirmado por Covid-19 en Costa Rica. <https://www.ministeriodesalud.go.cr/index.php/centro-de-prensa/ noticias/741-noticias-2020/1555-caso-confirmado-por-covid-19-en-costarica\#: : :text=06\%20de\%2oMarzo\%2ode\%202020, un\%2ohospedaje\%20 de\%20San\%20Jos\%C3\%A9.> [Consulta: 17 diciembre 2020].

"Escuela de Bibliotecología y Ciencias de la Información [EBCI]. 2020. Programa del curso Bl-1001 Técnicas de Investigación Bibliográfica. San José, Costa Rica: Universidad de Costa Rica.

" Hernández-Sampieri, Roberto y Christian Mendoza Torres. 2018. Metodología de la investigación: Las rutas cuantitativa, cualitativa y mixta. México: McGraw-Hill.

» Instituto Internacional de la UNESCO para la Educación Superior en América Latina y el Caribe. 2020. COVID-19 y educación superior: de los efectos inmediatos al día después; análisis de impactos, respuestas políticas y recomendaciones. Paris: Unesco IESALC. < https://unesdoc.unesco.org/ark:/48223/pfoooo375125 > [Consulta: 19 diciembre 2020].

»Kikut, Lorena. 2020. Análisis de resultados de la evaluación de la virtualización de cursos en la UCR ante la pandemia por Covid-19: perspectiva estudiantil. San José, Costa Rica: Centro de Evaluación Académica. Universidad de Costa Rica. <https://www.cea.ucr.ac.cr/images/stea/Eval_Doc/Analisis_ Virtualizacion_1-2020.pdf> [Consulta: 19 diciembre 2020].

" Moreira-Segura, Cristian y Brenda Delgadillo-Espinoza. 2015. La virtualidad en los procesos educativos: reflexiones teóricas sobre su implementación. En Tecnología en marcha. Vol. 28, No. 1, 121-129. < https://dialnet.unirioja.es/servlet/ articulo? codigo $=5051536>$ [Consulta: 17 diciembre 2020].

» Organización de las Naciones Unidas para la Educación, la Ciencia y la Cultura [UNESCO]. 2020. Covid-19 y educación superior: El camino a seguir después de la pandemia. <https://www.un.org/es/impacto-acad\%C3\%Agmico/covid-19-yeducaci\% $\mathrm{C}_{3} \% \mathrm{~B}_{3}$-superior-el-camino-seguir-despu\% $\mathrm{C}_{3} \%$ A9s-de-la-pandemia> [Consulta: 16 diciembre 2020].

»OREALC/UNESCO Santiago. 2020. Seminario web $n^{\circ} 11$ (A. Latina): COVID-19 y la Educación Superior. Impacto y recomendaciones. <https://www.youtube.com/ watch? $\mathrm{v}=\mathrm{zMmb}$ WWhYxk> [Consulta: 16 diciembre 2020].

"Rodríguez Valerio, Daniela. 2020. Más allá de la mensajería instantánea: WhatsApp como una herramienta de mediación y apoyo en la enseñanza de la Bibliotecología. En Información, cultura y sociedad. No. 42, 107-126. <https://doi. org/10.34096/ics.i42.7391> 
"Universidad de Costa Rica. Vicerrectoría de Docencia. 2020. Resolución VD-11502-2020: Lineamientos académicos y administrativos para la docencia con componente virtual. <http://vd.ucr.ac.cr/wp-content/uploads/2020/o9/ Resoluci\%C3\%B3n-VD-11502-2020.pdf> [Consulta: 13 diciembre 2020].

»Universidad de Costa Rica. Vicerrectoría de Investigación. 2020. Resolución VI7-2020. <https://vinv.ucr.ac.cr/sites/default/files/files/Resoluci\%C3\%B3n\%20 VI-7-2020.pdf> [Consulta: 10 diciembre 2020].

»Vindas, Manrique. 2020a. Rectoría de UCR anunció medidas obligatorias para prevenir contagio de coronavirus. <https://vinv.ucr.ac.cr/es/noticias/rectoria-deucr-anuncio-medidas-de-obligatorias-para-prevenir-contagio-de-coronavirus> [Consulta: 7 diciembre 2020].

»Vindas, Manrique. 2020b. La Vicerrectoría de Investigación crea normativa para la defensa virtual de trabajos finales de graduación. <https://www.ucr.ac.cr/ noticias/2020/05/27/la-vicerrectoria-de-investigacion-crea-normativa-para-ladefensa-virtual-de-trabajos-finales-de-graduacion.html>[Consulta: 13 diciembre 2020]. 